\title{
Singlet-Triplet Superconducting Quantum Magnetometer
}

\author{
Mohammad Alidoust,,${ }^{1, *}$ Klaus Halterman, ${ }^{2, \dagger}$ and Jacob Linder ${ }^{1, \ddagger}$ \\ ${ }^{1}$ Department of Physics, Norwegian University of Science and Technology, N-7491 Trondheim, Norway \\ ${ }^{2}$ Michelson Lab, Physics Division, Naval Air Warfare Center, China Lake, California 93555
}

(Dated: June 23, 2021)

\begin{abstract}
Motivated by the recent experimental realization of a quantum interference transistor based on the superconducting proximity effect, we here demonstrate that the inclusion of a textured ferromagnet both strongly enhances the flux sensitivity of such a device and additionally allows for singlet-triplet switching by tuning a bias voltage. This functionality makes explicit use of the induced spin-triplet correlations due to the magnetic texture. Whereas the existence of such triplet correlations is well-known, our finding demonstrates how spin-triplet superconductivity may be utilized for concrete technology, namely to improve the functionality of ultra-sensitive magnetometers.
\end{abstract}

PACS numbers: 74.50.+r, 74.45.+c, 74.25.Ha, 74.78.Na

\section{INTRODUCTION}

The synthesis of magnetism and superconductivity (SC) is intriguing both on a fundamental level and in terms of possible new devices based on the interplay between these phenomena. The former aspect encompasses a wide range of areas in condensed matter physics ranging from heavy-fermion compounds with several coexisting long-range orders ${ }^{1}$ to unusual forms of SC emerging in hybrid structures, displaying a resilience towards pair-breaking from impurities and paramagnetic limitations ${ }^{2,3}$. On the other hand, the Superconducting Quantum Interference Device ${ }^{4}$ is a prominent example of how the interaction between magnetism and SC may give rise to important functionality in technology $y^{5-7}$.

In a broader context, the emergence of multiple spontaneously broken symmetries is pivotal since it lies at the heart of a variety of phenomena outside the field of condensed matter. By creating heterostructures of ferromagnets (FM) and superconductors (S), such a situation is obtained due to the proximity effect ${ }^{8,9}:$ the induction of superconducting correlations in a magnetic material and vice versa ${ }^{12-15}$. One particular manifestation of the proximity effect emerges in an inhomogeneous FM which generates spin-polarized Cooper pairs with a large penetration depth despite the exchange field ${ }^{13,16}$. Whereas the existence of such spin-triplet superconducting correlations is well-known, it has remained unclear if they may be utilized for functional purposes in technology.

In this paper, we show that such proximity-induced spintriplet correlations can be utilized to obtain an ultra-sensitive interferometer with a highly stable flux-sensitivity that utilizes singlet and triplet proximity effects, both controlled via a bias voltage. We will refer to this device as a Singlet-Triplet Superconducting Quantum Magnetometer (SQM). The most important aspect is that it greatly enhances the range over which there is a flux-sensitivity compared to previously experimentally realized magnetometers ${ }^{5,6}$. One merit of this finding is that it demonstrates how spin-triplet SC may be utilized for a concrete technology, namely to improve the functionality of ultra-sensitive magnetometers which could lead to advancements in low temperature nanoscale spintronics that require minute flux detection. A similar interferometer was experi- mentally realized in ${ }^{5,6,18,19}$, albeit without any ferromagnetic element. Incorporating a textured ferromagnet in this type of device should therefore be feasible, and hence would allow for a test of our predictions. In addition, the considerable improvement in sensitivity in turn presents new possibilities for many practical applications spanning numerous disciplines, including medicine (neuromagnetic studies of the brain), geophysics (where field mapping is needed with high precision), and components of magnetoresistive devices (including memory cells).

We will demonstrate that the inclusion of an inhomogeneous FM having a spatially dependent magnetic texture, considerably improves the functionality of such an interferometer. In fact, the SQM features a stable phase-sensitivity which should be contrasted with the scenario without any ferromagnetic elements considered previously, in which case the sensitivity is finite only for special values of the external flux. As we will explain in detail, this effect originates from the triplet correlations present in the junction. To make our discussion as general as possible, we cover both the diffusive and ballistic regimes of transport, utilizing the Keldysh-Usade ${ }^{17}$ and Bogoliubov-de Gennes (BdG) ${ }^{8}$ formalisms.

\section{THEORY}

Assuming an applied voltage difference, $V$, between the non-superconducting region and the collector electrode (see Fig. 1), and neglecting Coulomb blockade effects, the resistive quasiparticle current is given by the following relation ${ }^{18,20,21}$ :

$$
\begin{aligned}
e R I_{q p} & =\frac{1}{W} \int_{-W / 2}^{+W / 2} \int_{-\infty}^{\infty} d x d \varepsilon N(x, \varepsilon, \phi, T) N_{S}(\varepsilon-e V) \\
& \times(f(\varepsilon-e V)-f(\varepsilon)),
\end{aligned}
$$

where $N(x, \varepsilon, \phi, T)$ is the local density of states (DOS) in the sandwiched region normalized by its value in the normal state and $N_{S}$ is the collector electrode's DOS which can be expressed by

$$
N_{S}(\varepsilon)=\operatorname{Re}\left\{|\varepsilon| / \sqrt{\varepsilon^{2}-\Delta(T)^{2}}\right\}
$$




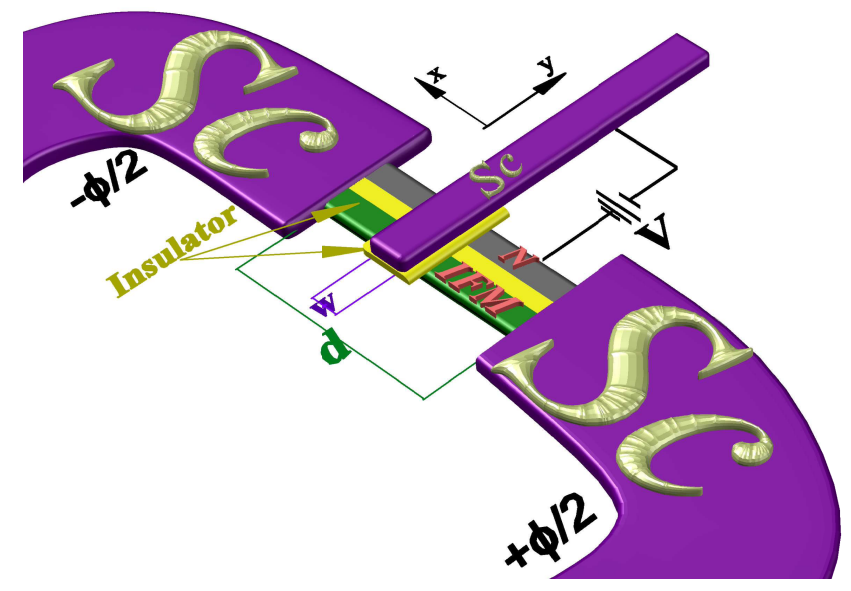

FIG. 1: Schematic set up of the proposed SQM. An inhomogeneous FM and a normal-metal (N) nanowire each of length $d$ are connected in parallel with an insulating layer separating them. This structure is sandwiched between two bulk $s$-wave superconductors along the $x$ axis. A third S electrode of width $W$ is deposited on top of the nanowires along the $y$-direction. By applying a voltage $V$, a quasiparticle current flows between the electrode and nano-wires (we refer to the third superconducting electrode as the "collector electrode"). The bulk $\mathrm{S}$ are assumed to be part of a closed loop circuit threaded by an external flux, so that any supercurrent flowing into the collector electrode can be neglected. The external flux induces a macroscopic phase difference, $\phi$, between the bulk S.

in the $s$-wave case. Here, $f(\varepsilon)$ is the Fermi-Dirac energy distribution, $\varepsilon$ is quasiparicle energy measured from the Fermi level, $\phi$ is the superconducting phase difference between the bulk superconducting leads, $e$ is the unit electronic charge, and $R$ is the resistance of the non-superconducting nano-wire. The integration is taken over the collector electrode width in the $x$-direction (see Fig. 1). We note in passing that if a voltage difference is present between the $\mathrm{S}$ banks, it is possible to induce Shapiro steps even in the absence of external radiation ${ }^{26}$. In our setup, however, no voltage shift exists between the bulk $\mathrm{S}$ regions.

To be concrete, we consider a conical texture for the FM and adopt a model relevant for Ho where the magnetic moment rotates on the surface of a cone with apex angle $\alpha=4 \pi / 9$ and turning angle $\varpi=\pi / 6$. If we assume that the distance of interatomic layers is $a=0.02 d_{F}{ }^{22}$, the exchange field, $\mathbf{h}$, can be written as $\mathbf{h}=h(\cos \alpha \hat{x}+\sin \alpha[\sin (\varpi x / a) \hat{y}+$ $\cos (\varpi x / a) \hat{z}])$. In the diffusive regime, the mean free path is much smaller than junction length ${ }^{17}$. In what follows, we consider the full proximity effect of the diffusive regime without making any simplifying assumptions such as low interface transparency or linearization of the equations. We emphasize that our BdG approach leads to the same results in the ballistic limit. The Usadel equation ${ }^{17}$ can be compactly written as

$$
D[\hat{\partial}, \hat{G}[\hat{\partial}, \hat{G}]]+i\left[\varepsilon \hat{\rho}_{3}+\operatorname{diag}\left[\mathbf{h} \cdot \underline{\sigma},(\mathbf{h} \cdot \underline{\sigma})^{\tau}\right], \hat{G}\right]=0 .
$$

Here $D$ is the diffusion constant, $\hat{G}$ represents the total Green's function and $\hat{\rho}_{3}$ and $\underline{\sigma}$ are $4 \times 4$ and $2 \times 2$ Pauli matrixes, respectively. The Usadel equation is supplemented by the Kupriyanov-Lukichev boundary conditions at interfaces along the $x$-axis ${ }^{23}$;

$$
2 \zeta \hat{G} \hat{\partial} \hat{G}=\left[\hat{G}_{\mathrm{BCS}}(\phi), \hat{G}\right],
$$

in which $\hat{G}_{\mathrm{BCS}}$ is the bulk solution and $\zeta$ controls interface opacity. To have more stability in numerical solutions, we use the so-called Ricatti parametrization of Green's function where the local DOS can be expressed by ${ }^{15,24}$ :

$$
N(x, \varepsilon, \phi, T)=\frac{1}{2} \operatorname{Re}\left\{\operatorname{Tr}\left[\left(1-\gamma^{R} \tilde{\gamma}^{R}\right) /\left(1+\gamma^{R} \tilde{\gamma}^{R}\right)\right]\right\} .
$$

To model realistic interfaces, we consider a finite barrier transparency by setting $\zeta=4$ : a perfect interface has $\zeta=0$ whereas the tunneling limit is reached for $\zeta \gg 1$. The value of $\zeta$ thus controls the magnitude of the proximity effect, but it has no bearing on our main result. We use $\hbar=k_{B}=1$ and set a fixed temperature throughout our computations equal to $0.05 T_{c}$. All lengths in the system are normalized by $\xi_{S}$, the superconducting coherence length.

\section{RESULTS AND DISCUSSION}

Since the underlying physical mechanism for the magnetometer is the modulation of the DOS in the nano-wire when changing the superconducting phase difference, we first show the results for this quantity in the top row of Fig. 2. The DOS is averaged over the width of the collector electrode and plotted vs. quasiparticle energy. The magnitude of the exchange splitting is set to $h=3 \Delta_{0}$ whereas the length of the nano-wires is set to $d=0.5 \xi_{S}$, which typically corresponds to $\sim 10 \mathrm{~nm}$. The columns correspond to (a) a S/N/S junction, (b) a S/uniform FM/S junction, and (c) a S/conical FM/S junction. For the $S / N / S$ junction in (a), we obtain the well-known minigap profile in the DOS spectrum ${ }^{15,18}$ whereas the minigap vanishes in a uniform FM junction shown in (b). This changes qualitatively for the conical FM junction in (c): the DOS now peaks near the Fermi level for some phase differences. This resonant behavior of the DOS in the inhomogeneous case is a manifestation of the presence of spin-triplet superconducting correlations which in the diffusive limit have an odd-frequency symmetry ${ }^{10,13}$.

To see how this influences the flux sensitivity, consider now the bottom rows of Fig. 2. Columns (a)-(c) correspond to the same type of junction as described above, and we have plotted the device sensitivity $d I_{q p} / d \phi$ as a function of the phase difference with $d=0.3 \xi_{S}$. The flux sensitivity can be directly related to the DOS shown in the top row of Fig. 2. To see this, consider first the normal and uniform FM cases in (a) and (b). The magnitude of $d I_{q p} / d \phi$ is highly suppressed for the uniform magnetization case for all values of voltage difference $e V / \Delta_{0}$ compared to normal junction. The superconducting correlations are effectively washed out due to destructive competition between singlet and short-range triplet correlations which suppresses the proximity effect overall, leading to a featureless DOS and resulting low device sensitivity ${ }^{24}$.

For the normal metal case, however, the flux-sensitivity is seen to feature a peak at a fixed phase. It should be noted that 

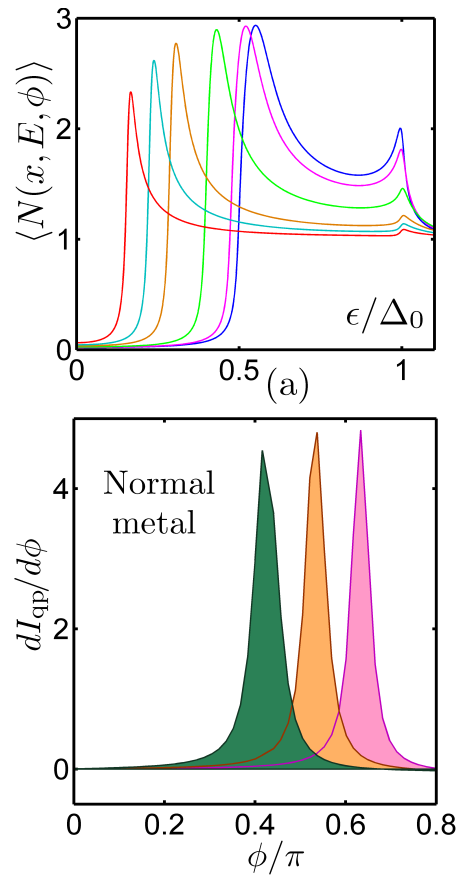
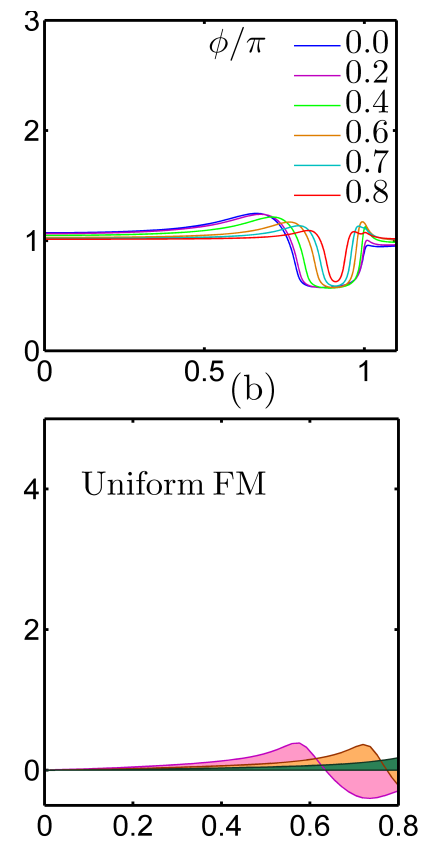
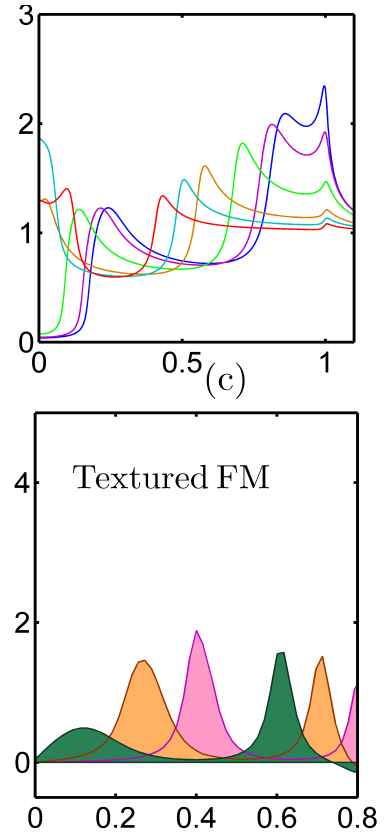

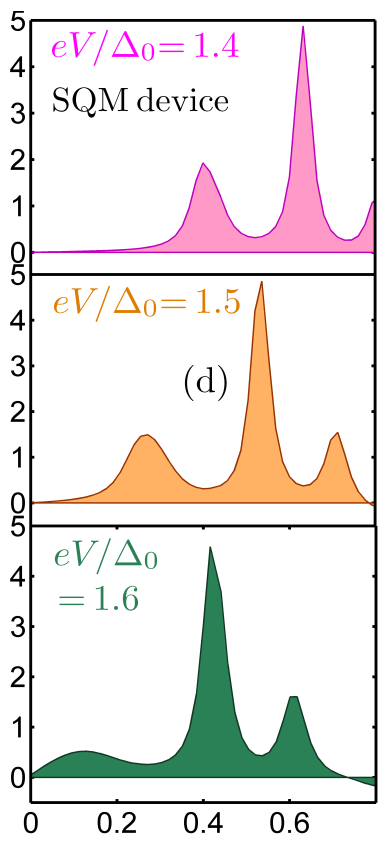

FIG. 2: (Color online) Top row (a)-(c): DOS vs. quasiparticle energy $\epsilon$ for several phase differences $\phi$. The columns correspond to (a) a S/N/S junction, (b) a S/uniform FM/S junction, and (c) a S/conical FM/S junction. The DOS has been averaged over the collector electrode width $W=d / 2$ and $d=0.5 \xi_{S}$. Bottom row (a)-(c): the device sensitivity $d I_{q p} / d \phi$ vs. phase difference $\phi$ for different choices of bias voltage $e V / \Delta_{0}=\{1.4,1.5,1.6\}$ with $d=0.3 \xi_{S}$. In the column (d), we show the sensitivity for our proposed SQM device which incorporates both a $\mathrm{N}$ and conical FM nano-wire (see Fig. 1).

a single peak always appears completely irrespective of the parameters chosen for the normal metal case. This means that whereas the sensitivity is high, it is restricted to a very narrow regime of external flux so that it would be insensitive to any magnetic fluxes deviating from this special value.

Consider now instead a junction with a conical FM shown in Fig. 2(c), where long-range spin-triplet correlations are present. The sensitivity $d I_{q p} / d \phi$ now shows a strongly enhanced stability with respect to $\phi$, which is present for several voltages. This can be clearly seen by noting the presence of two peaks in $d I_{q p} / d \phi$, which implies an improved device sensitivity by $\sim 100 \%$ compared to (a). Although the sensitivity amplitude is now somewhat smaller than the normal junction, the conical FM renders the magnetometer to have a flux-sensitivity covering a large part of the flux-regime. We will show below that this property used in conjunction with the singlet proximity effect from a normal metal produces a magnetometer with sensitivity for essentially any flux.

We first explain the physical origin of this enhanced fluxsensitivity arising from the presence of a textured FM. It is known that magnetic inhomogeneities can contribute to proximity induced triplet pairing in superconducting structures ${ }^{13}$. When this occurs, the DOS differs qualitatively from the nonmagnetic superconducting proximity effect. As seen in the top panel of Fig. 2(a), the the energy-resolved DOS for the nonmagnetic case simply exhibits a minigap, while in (c) a much stronger variation is observed. In particular, when the triplet correlations are present, a zero-energy peak arises rather than a gap as one tunes the superconducting phase difference $\phi$.
This can be traced back to the odd-frequency symmetry of the triplet correlations, which enhances the low-energy spectral weight. It is clear from the DOS of the textured FM case why the quasiparticle current is more sensitive to $\phi$ than in the previous $\mathrm{N}$ and uniform $\mathrm{F}$ cases: changing $\phi$ alters the DOS not only by simply closing a minigap, as in the $\mathrm{N}$ case, but by inducing a transition from a fully gapped to a peaked DOS at zero energy in addition to altering the spectral features at other subgap energies. By "gapped" we mean an absence of singleparticle states in the low-energy spectrum. The net result is a magnetometer sensitivity $d I_{q p} / d \phi$ which takes on appreciable values over a large interval of fluxes rather than only peaking near a specific value of the flux. These type of spin-polarized superconducting correlations in the system thus improves the device functionality.

Having now described how the presence of textured ferromagnetism alters the magnetometer sensitivity, we proceed to introduce the SQM device. Consider a situation where a $\mathrm{N}$ and a conical FM wire are connected in parallel as shown in Fig. 1. An insulating layer prevents interference between the two wires. When the connected wires are in electrical contact with a top superconducting electrode, a resistive quasiparticle current flows between them in the presence of an applied bias voltage $V$. We underline here that this resistive current is naturally separated from the supercurrent flowing in the superconducting loop due to current conservation. Let us denote the DOS of the $\mathrm{N}$ and conical FM regions by $N_{\mathrm{N}}$ and $N_{\mathrm{FM}}$. The energy-resolved quasiparticle current flowing to the top superconducting electrode will then be proportional to $N_{\mathrm{N}}+N_{\mathrm{FM}}$. 
The total current is obtained by integrating over all energies and taking into account the proper Fermi-Dirac distribution functions. The resultant flux-sensitivity, $d I_{q p} / d \phi$, of the proposed SQM is shown in Fig. 2(d).

Remarkably, the sensitivity is finite over almost the entire range of phase-differences, which means that the SQM would operate as a magnetometer over a much broader flux-range than in the experiments of Refs. 5,6,18. This advantage stems from the fact that the sensitivity $d I_{q p} / d \phi$ has its main contribution from different values of the phase (flux) in the $\mathrm{N}$ and conical FM cases, which is generated by the triplet correlations: the peaks in Fig. 2(a) and (c) occur at incommensurate values and therefore their combination yields a total sensitivity which covers essentially the entire range of fluxes and leads to $\mathrm{a} \sim 200 \%$ improvement of the device sensitivity. This idea can be extended to a device with several layers where an incommensurate sensitivity of each layer would generate a huge enhancement of the device efficiency. Another interesting aspect of the proposed device is that for longer junction lengths, the contribution to the sensitivity $d I_{q p} / d \phi$ from the singlet and triplet proximity effects occurs in different voltage regimes. For voltages close to $\mathrm{eV} \sim \Delta$, the singlet proximity effect dominates whereas for larger voltages $e V>1.5 \Delta$, the triplet proximity effect is responsible for the device sensitivity. This means that the contribution from the $\mathrm{N}$ and conical FM region can be individually separated by tuning the voltage $e V$, effectively switching the two wires "on" and "off".

We now turn to the ballistic regime of transport and utilize the BdG technique which enables us to fully isolate all triplet pairing correlations in the system and investigate the precise behavior of the proximity-induced triplet superconducting correlations, both for equal- and opposite spin-pairing. In terms of the quasiparticle amplitudes $u_{n}^{\sigma}$ and $v_{n}^{\sigma}$ with excitation energy $\varepsilon_{n}$, the $\mathrm{BdG}$ equations are written ${ }^{8,25}$ :

$$
\left(\begin{array}{cc}
\mathcal{H}_{M}-\mu \mathbb{I} & \Delta(x) \mathbb{I} \\
\Delta^{*}(x) \mathbb{I} & -\left(\mathcal{T} \mathcal{H}_{M} \mathcal{T}^{-1}-\mu \mathbb{I}\right)
\end{array}\right) \Psi_{n}=\varepsilon_{n} \Psi_{n}
$$

where the wavefunction reads

$$
\Psi_{n} \equiv\left(u_{n}^{\uparrow}, u_{n}^{\downarrow}, v_{n}^{\uparrow}, v_{n}^{\downarrow}\right)^{T}
$$

whereas the normal-state Hamiltonian is defined by:

$$
\mathcal{H}_{M}=-\vec{\nabla}_{x}^{2} / 2 m+\varepsilon_{\perp}-\mathbf{h} \cdot \underline{\sigma} .
$$

Above, $\mu$ is the chemical potential, and $\varepsilon_{\perp}$ represents the transverse kinetic energy. The time reversal operator, $\mathcal{T}$, is written $\mathcal{T}=i \sigma_{z} \mathcal{C}$, where $\mathcal{C}$ is the operator of complex conjugation. We take the pair potential, $\Delta(x)$, to be piecewise constant in the $\mathrm{S}$ regions with a phase difference $\phi$. To capture the triplet correlations, we align the quantization axis with the local magnetization vector in the conical FM.

The time-dependent triplet correlations with opposite $\left(f_{0}\right)$
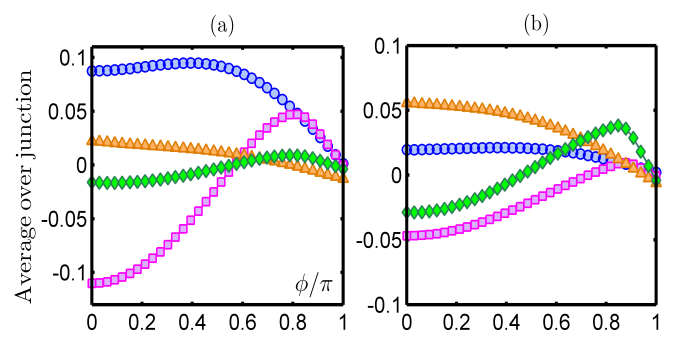

$\multimap \operatorname{Re}\left\langle f_{0}\right\rangle x$

$\longrightarrow \operatorname{Im}\left\langle f_{0}\right\rangle x$

$\triangle-\operatorname{Re}\left\langle f_{1}\right\rangle_{x}$

$\multimap \operatorname{Im}\left\langle f_{1}\right\rangle x$

FIG. 3: Imaginary and real parts of spin-triplet superconducting correlations averaged over the junction length plotted vs $\phi$ in the ballistic regime where a conical FM is sandwiched between two SC banks. (a) $d=0.3 \xi_{S}$ and (b) $d=0.5 \xi_{S}$.

and equal $\left(f_{1}\right)$ spin-pairings, are then expressed as:

$$
\begin{aligned}
f_{0}=\frac{1}{2} \sum_{n} & \left\{\cos \alpha f_{n}^{+}+\sin \alpha\left[\cos (\varpi x / a)\left(f_{n}^{\uparrow \downarrow}-f_{n}^{\downarrow \uparrow}\right)\right.\right. \\
+ & \left.\left.i \sin (\varpi x / a) f_{n}^{-}\right]\right\} \zeta_{n}(t), \\
f_{1}=\frac{1}{2} \sum_{n}\{ & \sin \alpha f_{n}^{+}-\cos \alpha\left[\cos (\varpi x / a)\left(f_{n}^{\uparrow \downarrow}-f_{n}^{\downarrow \uparrow}\right)\right. \\
+ & \left.\left.i \sin (\varpi x / a) f_{n}^{-}\right]\right\} \zeta_{n}(t),
\end{aligned}
$$

in which we define

$$
f_{n}^{ \pm} \equiv f_{n}^{\uparrow \uparrow} \pm f_{n}^{\downarrow \downarrow} ; \quad f_{n}^{\sigma \sigma^{\prime}} \equiv u_{n}^{\sigma} v_{n}^{\sigma^{\prime} *} .
$$

We also have introduced the time-dependent quantity ${ }^{14}$

$$
\zeta_{n}(t)=\cos \left(\varepsilon_{n} t\right)-i \sin \left(\varepsilon_{n} t\right) \tanh \left(\varepsilon_{n} /(2 T)\right) .
$$

When $t=0$, the triplet correlations vanish by virtue of the Pauli exclusion principle. As $t$ is increased, the triplet correlations generally form near the F/S interfaces, and slowly grow in amplitude. In what follows, we scale $t$ by a characteristic "Debye" energy, $\omega_{D}$, and set $\omega_{D} t=8.8$ as a representative choice which allows one to witness the extended behavior of the triplets in the junction. When the junction contains a conical FM, the equal spin-pairing components are generally nonzero and their evolution against phase difference is shown in Fig. 3. For $d=0.3 \xi_{S}$ (left panel), it is seen that the averaged opposite spin-pairing components are larger in magnitude and have the greatest variation with phase, whereas both types of triplet correlations feature a non-monotonic behavior vs $\phi$, nearly vanishing at $\phi=\pi$. As we increase the junction length to $d=0.5 \xi_{S}$ however, it is seen that the $f_{1}$ correlations are now more prominent. This is consistent with the fact that the equalspin correlations have a long-ranged penetration depth in spite of the presence of an exchange field, whereas the oppositespin triplet pairs have increased oscillations that decay over a length scale similar to that of the spin-singlet state, leading to reduced spatial averages.

\section{SUMMARY}

In summary, we have shown that long-ranged spin-triplet superconducting correlations can be utilized for practical 
technologies and offer advancements in the form of improved sensitivity for magnetometers. The key element is the inclusion of a textured ferromagnet and a normal-metal which alters the superconducting proximity effect and allows for a $\sim 200 \%$ increased flux-operation range for the proposed device. We demonstrate a highly stable flux-sensitivity that utilizes both singlet and triplet proximity effects. We found a considerable enhancement of the range over which there is a flux-sensitivity compared to previously fabricated magnetometers in which there are no ferromagnet elements.

\section{Acknowledgments}

K.H. is supported in part by IARPA and by a grant of supercomputer resources provided by the DOD HPCMP. J.L. was supported by the COST Action MP-1201 "Novel Functionalities through Optimized Confinement of Condensate and Fields".
* Electronic address: phymalidoust@gmail.com

$\dagger$ Electronic address: klaus.halterman@navy.mil

¥ Electronic address: jacob.linder@ntnu.no

1 S. S. Saxena, and P. B. Littlewood, Nature 406, 587 (2000); D. Aoki, A. Huxley, E. Ressouche, D. Braithwaite, J. Flouquet, J.-P. Brison, E. Lhotel, and C. Paulsen, Nature 413, 613 (2001).

2 V. L. Berezinskii, JETP Lett. 20, 287 (1974).

3 F. S. Bergeret, A. F. Volkov, and K. B. Efetov, Phys. Rev. Lett. 86, 4096 (2001).

${ }^{4}$ R. C. Jaklevic, J. Lambe, A. H. Silver, and J. E. Mercereau, Phys. Rev. Lett. 12, 159 (1964).

5 F. Giazotto, J. T. Peltonen, M. Meschke, and J. P. Pekola, Nature Physics 6, 254 (2010).

${ }^{6}$ F. Giazotto, P. Spathis, S. Roddaro, S. Biswas, F. Taddei, M. Governale, and L. Sorba, Nature Physics 7, 857 (2011).

${ }^{7}$ P. Spathis, S. Biswas, S. Roddaro, L. Sorba, F. Giazotto and F Beltram, Nanotechnology 22, 105201, (2011).

8 P. G. De Gennes, Rev. Mod. Phys. 36, 225 (1964).

9 W. L. McMillan, Phys. Rev. 175, 537 (1968).

${ }^{10}$ T. Kontos, M. Aprili, J. Lesueur, and X. Grison, Phys. Rev. Lett. 86, 304 (2001).

11 V.V. Ryazanov et al., J. Low Temp. Phys. 136, 385 (2004); V. A. Oboznov, V. V. Bolginov, A. K. Feofanov, V. V. Ryazanov, and A. I. Buzdin, Phys. Rev. Lett. 96, 197003 (2006).

12 A. I. Buzdin, Rev. Mod. Phys. 77, 935 (2005); M. Eschrig, Phys. Today 64(1), 43 (2011).

${ }^{13}$ F. S. Bergeret, A. F. Volkov, and K. B. Efetov, Rev. Mod. Phys. 77, 1321 (2005).

${ }^{14}$ K. Halterman, P. H. Barsic, and O. T. Valls, Phys. Rev. Lett. 99, 127002 (2007).
15 F. Zhou et al., J. Low Temp. Phys. 110, 841 (1998); J. C. Hammer, J. C. Cuevas, F. S. Bergeret, and W. Belzig, Phys. Rev. B 76, 064514 (2007).

16 J. W. A. Robinson, J. D. S. Witt, M. G. Blamire, Science 329, 5987 (2010); M. Alidoust, and J. Linder, Phys. Rev. B 82, 224504 (2010); G. B. Halasz, M. G. Blamire, and J. W. A. Robinson, Phys. Rev. B 84, 024517 (2011); C. Wu, O. T. Valls, and K. Halterman, Phys. Rev. Lett. 108, 117005 (2012).

17 K. D. Usadel,Phys. Rev. Lett. 25, 507 (1970); A. I. Larkin et al., in Nonequilibrium Superconductivity, edited by D. Langenberg and A. Larkin (Elsevier, Amesterdam, 1986), P. 493.

18 F. Giazotto, and F. Taddei, Phys. Rev. B 84, 214502 (2011).

19 M. Meschke, J. T. Peltonen, J. P. Pekola, and F. Giazotto, Phys. Rev. B 84, 214514 (2011).

${ }^{20}$ Likharev, Rev. Mod. Phys. 51, 101 (1979); A.A. Abrikosov, Fundamentals of the Theory of Metals, Amsterdam, Netherlands : North-Holland (1988).

21 A. S. Vasenko, S. Kawabata, A. A. Golubov, M. Yu. Kupriyanov, C. Lacroix, F. S. Bergeret, and F. W. J. Hekking, Phys. Rev. B 84, 024524 (2011).

${ }^{22}$ M. Alidoust, J. Linder, G. Rashedi, T. Yokoyama, and A. Sudb $\varnothing$, Phys. Rev. B 81, 014512 (2010).

23 A. V. Zaitsev, Zh. Eksp. Teor. Fiz. 86, 1742 (1984) (Sov. Phys. JETP 59, 1015 (1984); M. Y. Kuprianov et al., Sov. Phys. JETP 67, 1163 (1988).

${ }^{24}$ M. Alidoust, G. Rashedi, J. Linder, and A. Sudbø, Phys. Rev. B 82, 014532 (2010).

25 K. Halterman, and O. T. Valls, Phys. Rev. B 65, 014509 (2002).

${ }^{26}$ J. C. Cuevas, and H. Pothier, Phys. Rev. B 75, 174513 (2007). 